\title{
Analysis and Mathematical Description of the Ideal Thermotropic Cycle of Internal Combustion Engines
}

\author{
Mikhail Stolbov \\ Department of Heat Engines and Power Plants, Institute of Mechanical Engineering and Road Transport, VISU (Vladimir State University \\ Named After A. G. and N. G. Stoletovs), Vladimir, Russian Federation \\ Email address: \\ Mihai_Stolbov@mail.ru

\section{To cite this article:} \\ Mikhail Stolbov. Analysis and Mathematical Description of the Ideal Thermotropic Cycle of Internal Combustion Engines. Engineering and \\ Applied Sciences. Vol. 3, No. 4, 2018, pp. 97-102. doi: 10.11648/j.eas.20180304.11
}

Received: July 25, 2018; Accepted: September 18, 2018; Published: October 29, 2018

\begin{abstract}
A mathematical model of an ideal thermotropic cycle is proposed, which extends the idea of real working processes in the internal combustion engine (ICE). The thermotropic cycle the same for diesel, gasoline and gas ICE, and also provides significantly better correspondense to the real cycles than classical cycles. Heat supply to the cycle is carried out in the form of a combined process "compression-expansion". The combined process consists of two incomplete thermotropic processes and with a high degree of approximation reproduces the laws of real combustion processes in the ICE, from compression to expansion. The mathematical model of the basic thermotropic process is based on the laws of molecular kinetic theory and thermodynamics of ideal gases. The main thermotropic process is based on three fundamental equations of thermodynamics: differential equations of the first law of thermodynamics and the law of heat exchange of the external source of the working medium, as well as the equation of state (Clapeyron). The heat of the process in the thermotropic process, in contrast to the polytropic, is an independent value and takes into account fuel consumption. The equation of the main thermotropic process has additivity, which allows us to consider its parameters as the sum of the adiabatic and thermal components of the process occurring in the gas mixture. An important feature of the thermotropic process is also the variable heat capacity of the process. The gas laws of classical thermodynamics are special cases of a new process. The advantage of the new cycle model is mathematical simplicity. The initial system of equations made it possible to perform precise integration and express the equations of processes and cycles in elementary functions. Precise integration ensured high accuracy of estimation of influencing factors contained in the model and absolute convergence of thermal and material balances of the cycle. A number of elements of the theory of thermotropic processes and cycles can be used to replace isochoric-isobaricpolytropic combustion models in undergraduate and specialist studies. This is facilitated by the absence of the need for complex computer programs that significantly complicate the development of educational material. In General, models with more complex laws of heat supply can be useful for undergraduates and graduate students to pre-evaluate the effectiveness of the proposed project activities to improve the internal combustion engine.
\end{abstract}

Keywords: Thermotropic Thermodynamic Process, Thermotropic Process "Compression-Expansion", Thermotropic Ideal Cycle

\section{Introduction}

Courses of technical thermodynamics [1-5] and the theory of working processes of internal combustion engines [6 - 8] consider three ideal gas cycles: isochoric, isobaric and mixed. Due to the high degree of idealization, they do not adequately reflect the real processes in the engines. Therefore, a new thermodynamic model of an ideal thermotropic cycle was proposed, not only generalizing classical ideal cycles, but also providing a significantly higher degree of approximation to reality [9]. In this paper, we consider an additional possibility of approaching taking into account some features of the combustion process in diesel engines and spark ignition engines. 

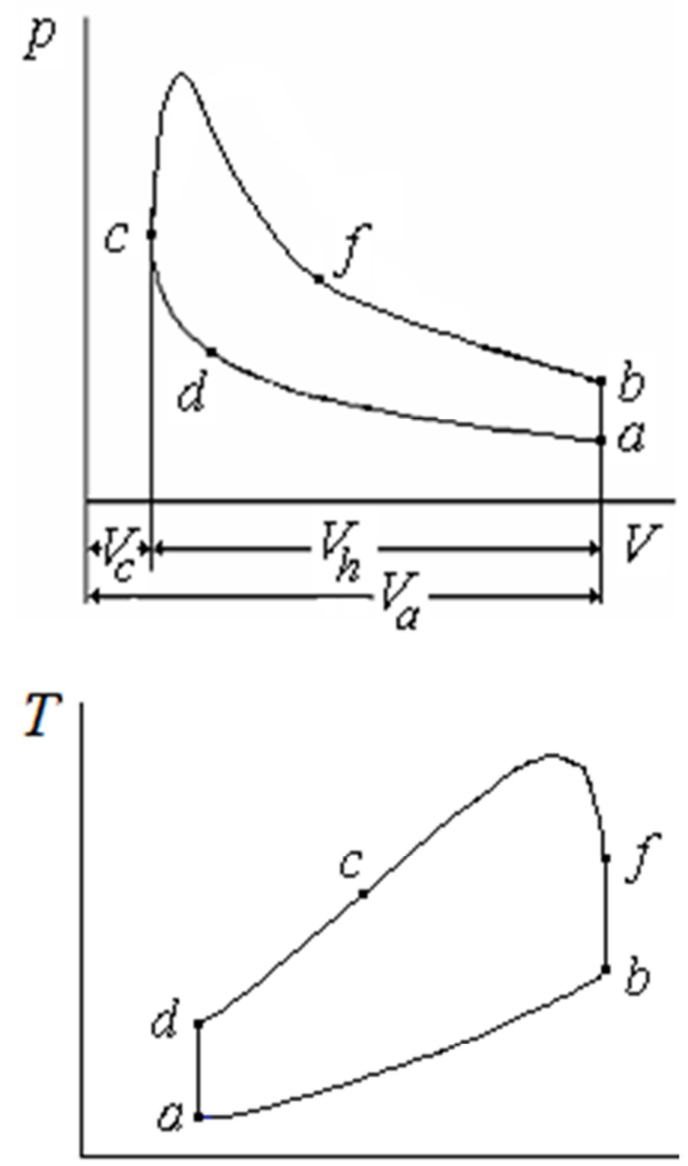

$S$

Figure 1. Diagram of the indicator diagram of an ideal thermotropic cycle in $p$ - $V$ and $T$-s coordinates.

\section{New Thermotropic Models of Processes and Cycle}

Figure 1 shows the $\mathrm{p}-\mathrm{V}$ diagram of an ideal closed thermotropic cycle. The heat exchange diagram (d-c-f curve) consists of a model of a combined thermotropic process "compression-expansion" refined in comparison with [4], two processes of adiabatic compression (a-d section) and expansion ( $\mathrm{f}-\mathrm{b}$ ), as well as an isochoric process of heat removal into a cold environment $(b-a)$.

\subsection{Thermotropic Thermodynamic Process}

The basis of the combined processes is a thermotropic process. It generalizes the classical thermodynamic processes (including the polytropic process) and is their development. The term "thermotropic" (Greek. therme - heat; tropos - turn, direction) is based on the fact that the main element in the mathematical models of new processes is an analytically defined law of heat exchange between the external source and the working medium.

Thermotropic and polytropic equations are obtained by integrating a system of three equations. The first two equations are the same for both processes - the differential equation of the first law of thermodynamics and the equation of Clapeyron. However, in the polytrope in order to provide mathematical simplicity $[10,11]$, the third equation is $d Q=$ $M c_{\text {pol }} d T$, where $M$ is the quantity working medium, $Q$ is the current heat, $c_{\text {pol }}$ is the heat capacity of the process, taken as a constant, $T$ is the current temperature of the gas.

As a result, the heat of the polytropic process $Q_{0 \text { pol }}$ is determined by the process parameters: the value of $c_{\text {pol }}$, the exponent of $n$ and the initial and final temperatures of the gas. This is a serious drawback of the polytropic model, since in the real engine the heat of the $Q_{0}$ process depends only on the amount of fuel.

In the thermotropic process the third is the equation $d Q=$ $Q_{0} d x$, where $Q_{0}$ is the heat of the process, $x$ is the dimensionless law of heat exchange (figure 2) of the working medium with the environment. In this case, $Q_{0}$ is an independent value, and $x$ is equal to the change in the $Q / Q_{0}$ ratio during the process. For the thermotropic process, it is assumed that $x=f\left(v^{\mathrm{r}}\right)$, where $v^{\mathrm{r}}$ is a typical power function for the laws of the thermodynamic gas, and that the boundary conditions $\mathrm{x}=0$ for $v=v_{1}$ and $x=1$ for $v=v_{2}$. Then

$$
x=Q / Q_{0}=\left(v^{1-m}-v_{1}^{1-m}\right)\left(v_{2}^{1-m}-v_{1}^{1-m}\right),
$$

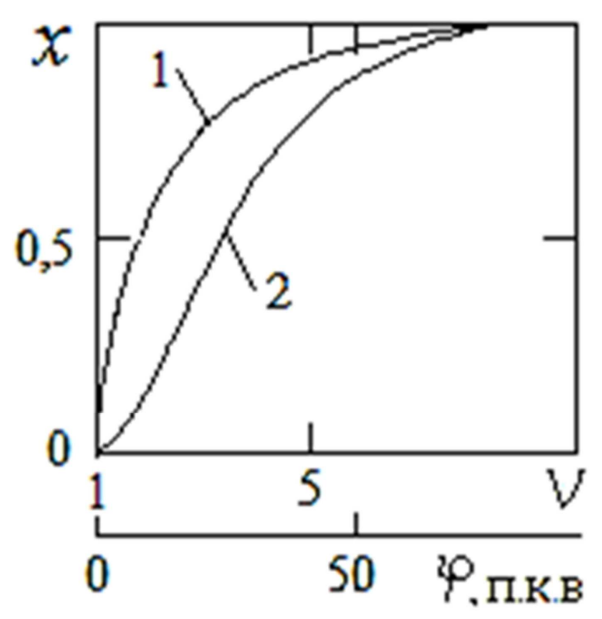

Figure 2. Changing the calculated laws of heat transfer $x$ depending on the volume (line 1) and time (crankshaft angle) (line 2) at $m=4$ and $v_{2} / v_{1}=8$.

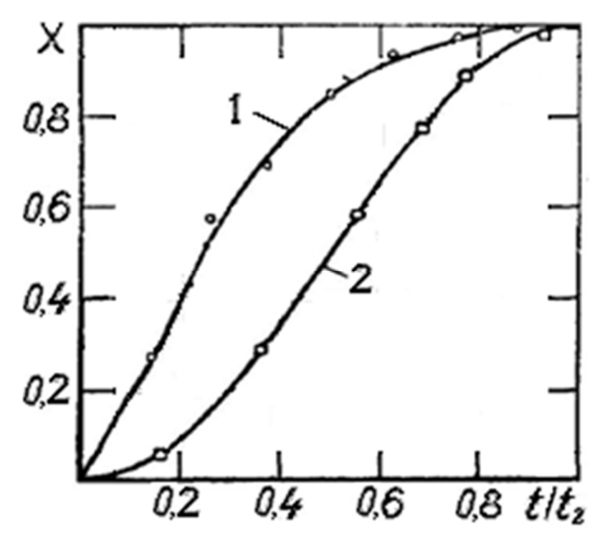

Figure 3. Experimental curves of heat release during the combustion fuel depending on time: 1-aviation diesel; 2- spark ignition engine; $t / t_{z}-$ relative time. 
where $v$ is the specific volume related to the mass $M$, indices 1 and 2 mean the initial and final volumes of gas, the absence of the index - to the current volume, $m=-r$ is an exponent of the thermotropic process.

The comparison of $x$ in figures (2) and (3) showed that, despite some discrepancy in the configurations of the same characteristic $\mathrm{X}$ in the coordinates of the relative volume $v / v_{1}$ [12] and the time $t / t_{1}$ [13], curves 1 and 2 in figure 2 satisfactorily display the experimental curves [14].

The analysis showed that the function $x_{\mathrm{pol}}=Q_{\mathrm{pol}} / Q_{0 \mathrm{pol}}$ ( $Q_{\mathrm{pol}}$ and $Q_{0 \mathrm{pol}}$ - current heat and heat of the polytropic process) is $x$ by the formula (1) at $n=m$. This means that the laws of $x$ and $x_{\text {pol }}$ are equal. The equality of the heat exchange law of the polytropic process and the analytical law of heat exchange (1) indicates a high degree of continuity of the latter with respect to classical thermodynamics.

The equation of the thermotropic process is obtained after the first law of thermodynamics equation is introduced the first derivative of the function (1) and integrates a system of three equations [15 - 18].

The current gas pressure in the thermotropic process is found by the formula,

$$
p=p_{1}\left[\left(1-A_{T}\right)\left(v / v_{1}\right)^{-k}+A_{T}\left(v / v_{1}\right)^{-m}\right]
$$

where $A_{\mathrm{T}}$ is a dimensionless constant coefficient for the process:

$$
A_{T=} q_{0}(1-m)\left\{c_{v} T_{1}(k-m)\left[\left(v_{2} / v_{1}\right)^{1-m}-1\right]\right\}^{-1},
$$

$p_{1}$ and $T_{1}$ is the initial pressure and temperature; $q_{0}$ is the specific heat of the process; $c_{\mathrm{v}}$ - isochoric heat capacities of the working medium, independent of the ideal cycle of the temperature, $k=c_{\mathrm{p}} / c_{\mathrm{v}}$ - the ratio of isobaric and isochoric heat capacities of the working medium (adiabatic coefficient).

The current temperature in the thermotropic process is as follows

$$
\begin{gathered}
T=T_{1}\left[\left(1-A_{T}\right)\left(v / v_{1}\right)^{1-k}+A_{T}\left(v / v_{1}\right)^{1-m}\right]= \\
=T_{1}\left(p / p_{1}\right)\left(v / v_{1}\right) .
\end{gathered}
$$

Analysis of formula (2) and (3) showed the following:

- pressure and temperature in the thermotropic process are the sum of two partial components - adiabatic and thermal;

- thermotropic process refers to processes with variable specific heat of the $c_{\mathrm{T}}$ process at an independent value of the heat of the $Q_{0}$ process;

- the value of the $A_{\mathrm{T}}$ - the ratio the thermotropic heat $Q_{0}$ to the polytropic heat $Q_{0 \text { pol }}$ at $m=n$;

- polytropic process is a special case of thermotropic process at $A_{\mathrm{T}}=1, m=n$.

The selected lines $\left(A_{\mathrm{T}}=1\right)$ on the figure 4 belong to the polytropic process and are known in the theory of thermodynamics. At $A_{\mathrm{T}} \neq 1$ line ratio $c_{\mathrm{T} 1} / c_{\mathrm{v}}$ depending on the absolute value and the sign of $A_{\mathrm{T}}$ flow similar to the lines of the polytropic process. The discontinuities function $c_{\mathrm{T} 1} / c_{\mathrm{V}}$ corresponds to the maximum temperature process. The location of the lines in the entire field of the graph means that the model of the thermotropic process corresponds to a much larger number of real thermodynamic processes than the polytropic one.

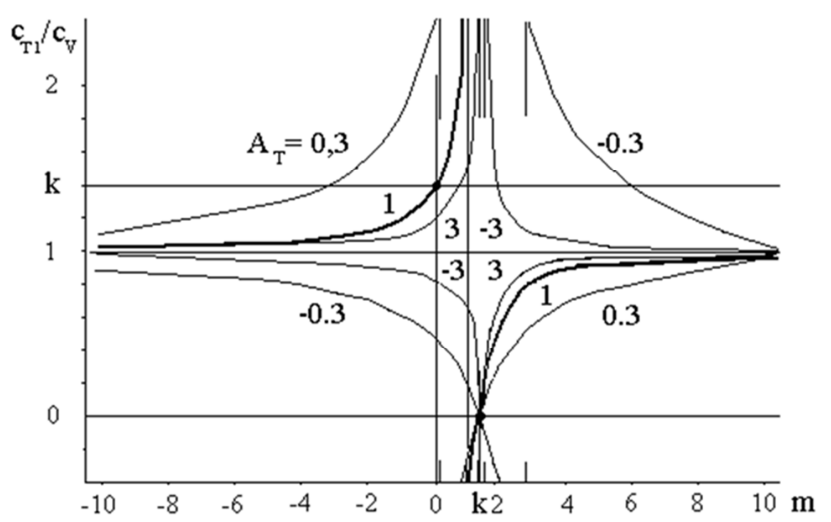

Figure 4. The dependence of the relative heat capacity at the initial point of the thermotropic process on $m$ at different values of the parameter $A_{T}$.

Relative volumes at maximum pressure and maximum temperature are determined by the following expressions:

$$
\begin{gathered}
v_{\text {pmax }} / v_{1}=\left[(k / m)\left(1-A_{T}^{-1}\right)\right]^{(k-m)^{-1}}, \\
v_{\text {Tmax }} / v_{1}=\left[(k-1)(m-1)^{-1}\left(1-A_{T}^{-1}\right)\right]^{(k-m)^{-1}} .
\end{gathered}
$$

The presence of maximum temperatures and pressures during expansion distinguishes thermotropic processes from polytropic ones and allows modeling gas processes with fuel combustion with sufficient approximation.

The current specific work of the thermotropic process is determined by the expression

$$
l=R T_{1}\left\{\begin{array}{c}
\left(1-A_{T}\right)(1-k)^{-1}\left[\left(v / v_{1}\right)^{1-k}-1\right]+ \\
+A_{T}(1-m)^{-1}\left[\left(v / v_{1}\right)^{1-m}-1\right]
\end{array}\right\}
$$

\subsection{Thermotropic Thermodynamic the Process of "Compression-Expansion"}

The combined "compression-expansion process" is a continuous heat transfer thermotropic process that starts with compression and ends with expansion. Thermotropic processes in this case are incomplete. The condition for the transition of the compression process in the process of expansion is the equality of the values of the functions $x_{\mathrm{compr}}$ $=x_{\exp }$ in the expression (1) and their first derivatives $(\mathrm{d} x / \mathrm{d} v)_{\text {compr }}=(\mathrm{d} x / \mathrm{d} v)_{\exp }$ in the point of transition from compression to expansion $c$ (figura 1).

In the case of using the "compression-expansion" process according to figure 1 , the following symbols of specific volumes and their ratios are used: $v$ - current volume, $v_{c}$ volume of transition from compression to expansion (point $c$ ), $v_{\mathrm{d}}$ and $v_{\mathrm{f}}$ - volumes at the beginning (point $d$ ) and at the end (point $f$ ) of heat dissipation; $\theta=V_{\mathrm{d}} / V_{\mathrm{c}}$ and $\rho=V_{\mathrm{f}} / V_{\mathrm{c}}$ - relative volumes at the beginning and at the end of heat supply.

Unlike [18], we consider thermotropic processes with different exponents of $m$. The equations $x$ in this case have the form: 
- under compression on the $d-c$ section

$$
x_{s}=x_{c}\left[\left(v / v_{d}\right)^{1-m_{s}}-1\right]\left(\theta^{m_{s}}-1\right)^{-1},
$$

- under expanding on the $c-f$ section

$$
x_{r}=x_{c}+\left(1-x_{c}\right)\left[\left(v / v_{c}\right)^{1-m_{r}}-1\right]\left(\rho^{1-m_{r}}-1\right)^{-1} .
$$

Here values $x_{\mathrm{s}}$ and $x_{\mathrm{r}}$ are the $x$ values at points $c, m_{\mathrm{s}}$ and $m_{\mathrm{r}}$ - exponents in sections $d-c$ and $c-f$.

Figure 5 shows that the application of these conditions allows the functions $x=\mathrm{F}(v)$ to provide a fairly good match between the calculated [18] and experimental [19] heat dissipation lines in the compression and expansion areas for different engines. When processing a real indicator chart, it is more convenient to first define $v_{\mathrm{d}}, x_{\mathrm{c}}$ and $m_{\mathrm{r}}$. In this case, the exponent $m_{\mathrm{s}}$ is determined by solving the implicit equation

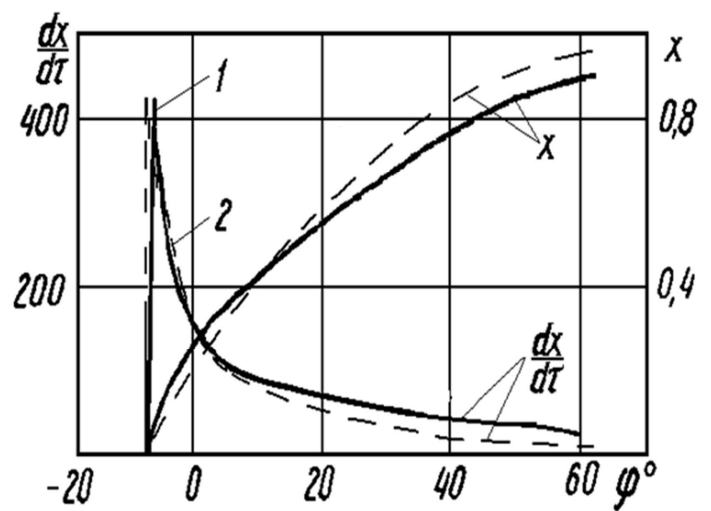

a)

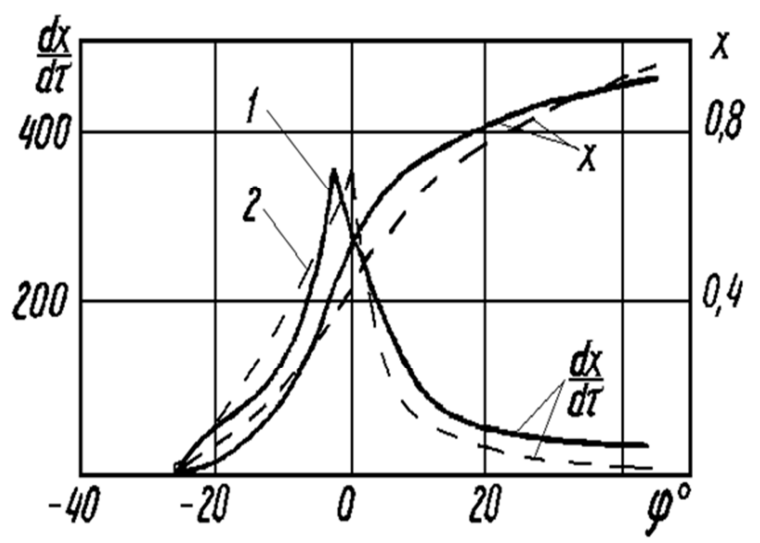

b)

Figure 5. Experimental and calculated laws of fuel combustion in compression and expansion depending on the rotation angle of the engine shaft: $a)$ in a diesel engine $\left(m_{s}=-11.6, m_{r}=4.3\right)$, b) in a spark ignition engine $\left(m_{s}=10.3, m_{r}=7.2\right)$; 1-experimental lines, 2-calculation lines on (7)- (8).

$$
\left(m_{s}-1\right)\left(1-\theta^{1-m_{s}}\right)^{-1}=\left(1 / x_{c}-1\right)\left(1-m_{r}\right)\left(\rho^{1-m_{r}}-1\right)^{-1} .
$$

The $m_{\mathrm{r}}$ value in the combined process for different engines can be from 2 to 7 .

As a result, the equations of the current values of pressure and temperature in the field of thermotropic compression are obtained $d-c$

$$
p_{s}=p_{1}\left[\left(1-A_{T S}\right)\left(v / v_{d}\right)^{-k}+A_{T S}\left(v / v_{d}\right)^{-m_{s}}\right],
$$

where the parameter

$$
\begin{gathered}
A_{T s}=\frac{x_{s} q_{0}\left(1-m_{s}\right)}{c_{v} T_{1}\left(k-m_{s}\right)\left(\theta^{m_{s}-1}-1\right)}, \\
T_{s}=T_{1}\left[\left(1-A_{T s}\right)\left(v / v_{d}\right)^{1-k}+A_{T s}\left(v / v_{d}\right)^{1-m_{s}}\right] .
\end{gathered}
$$

Equation of pressure and temperature in the area thermotropic of the $c-f$ has the form

$$
p_{r}=p_{c}\left[\left(1-A_{T r}\right)\left(v / v_{c}\right)^{-k}+A_{T r}\left(v / v_{c}\right)^{-m_{r}}\right],
$$

where the parameter

$$
\begin{gathered}
A_{T r}=\frac{\left(1-x_{c}\right) q_{0}\left(1-m_{r}\right)}{c_{v} T_{c}\left(k-m_{r}\right)\left[\rho^{\left.1-m_{r}-1\right]}\right.}=-A_{T s} \frac{p_{1}\left(k-m_{s}\right) \theta^{m_{s}}}{p_{c}\left(k-m_{r}\right)}, \\
T_{r}=T_{c}\left[\left(1-A_{T r}\right)\left(v / v_{c}\right)^{1-k}+A_{T r}\left(v / v_{c}\right)^{1-m_{r}}\right]
\end{gathered}
$$

Pressure $p_{\mathrm{c}}$ and temperature $T_{\mathrm{c}}$ in (13) and (15) for the area $c-f$ is determined by (10) and (12) for the volume $v=v_{\mathrm{c}}$.

The maximum values pressure and temperature in the process of "compression-expansion" are determined by the (4) and (5) with $v_{1}=v_{\mathrm{c}}, m=m_{\mathrm{r}}$ and $A_{\mathrm{T}}=A_{\mathrm{Tr}}$.

Current specific work of the process "compressionexpansion" in the cycle according to the scheme figura 1 determined on the basis of (6) and (10) - (15):

- in the compression zone $v_{d}-v_{c}$ - when using $T_{1}=T_{d}, v_{1}=$ $v_{d}, m=m_{\mathrm{s}}$ и $A_{\mathrm{T}}=A_{\mathrm{Ts}}$;

- in the expansion zone $v_{\mathrm{c}}-v_{f}$ - when using $T_{l}=T_{c}, v_{1}=v_{\mathrm{c}}$, $m=m_{\mathrm{r}}$ и $A_{\mathrm{T}}=A_{\mathrm{Tr}}$. The work of the $l_{\Sigma \mathrm{s}}$ thermotropic processes on the $d$-c section and $l_{\Sigma \mathrm{r}}$ on the $c-f$ section is performed after substitution $v=v_{\mathrm{c}}$ and $v=v_{f}$. The total work "compressionexpansion" in the $d-f$ section is equal to

$$
l_{\Sigma}=l_{\Sigma s}+l_{\Sigma r} .
$$

\subsection{Indicators of the Thermotropic Cycle}

The thermal efficiency of the cycle in the application of the process" compression - expansion " is determined by the dependence

$\eta_{t}=1-\frac{1}{\varepsilon^{k-1}}\left(\frac{\theta^{k-m_{S}-1}}{k-m_{S}}+\frac{\rho^{k-m_{r}}-1}{k-m_{r}}\right)\left(\frac{\theta^{1-m_{S-1}}}{1-m_{S}}+\frac{\rho^{1-m_{r}-1}}{1-m_{r}}\right)^{-1}$,

where $\varepsilon$ is the compression ratio.

It follows from (17) that $\eta_{t}$ is determined by the compression ratio $\varepsilon$, the heat capacity of the working medium $c_{\mathrm{T}}$, the exponent of the degree of the process "compressionexpansion" $m_{\mathrm{s}}$ and $m_{\mathrm{r}}$, as well as the location of the process relative to the point $c$, characterized by the parameters $\theta$ and $\rho$ (Figure 1).

The maximum thermal efficiency is found at the optimal $\theta_{\eta}$ value determined by solution the implicit equation:

$$
\begin{gathered}
\theta_{\eta}^{k-1}-\frac{1}{\varepsilon^{k-1}}\left(\frac{\theta^{k-m_{s}-1}}{k-m_{s}}+\frac{\rho^{k-m_{r}}-1}{k-m_{r}}\right)\left(\frac{\theta^{1-m_{s}-1}}{1-m_{s}}+\right. \\
\left.\frac{\rho^{1-m_{r}-1}}{1-m_{r}}\right)^{-1}=0 .
\end{gathered}
$$


When $\theta \neq \theta_{\eta}$ and the loop is configured for maximum efficiency it is necessary to adjust the value of $m_{\mathrm{s}}$ according to (9) with $\theta=\theta_{\eta}$ and clarify the value of $\eta_{\mathrm{t}}$ in (17).

The average cycle pressure is found by the formula

$$
p_{t}=\frac{q_{0} p_{a} \varepsilon}{R T_{a}(\varepsilon-1)} \eta_{t}
$$

Important indicators of the real engine cycle are the average and maximum rate of pressure change during fuel combustion [11].

It is recommended to determine the speeds in the same way as in the processing of the indicator diagrams of the real engine - according to the generally accepted formula

$$
\Delta p / \Delta \varphi=\left(p_{2}-p_{1}\right) /\left(\varphi_{2}-\varphi_{1}\right),
$$

where $p$ - is pressure, $\varphi$ - is the angle of rotation of the crankshaft. The average pressure increase rate is determined

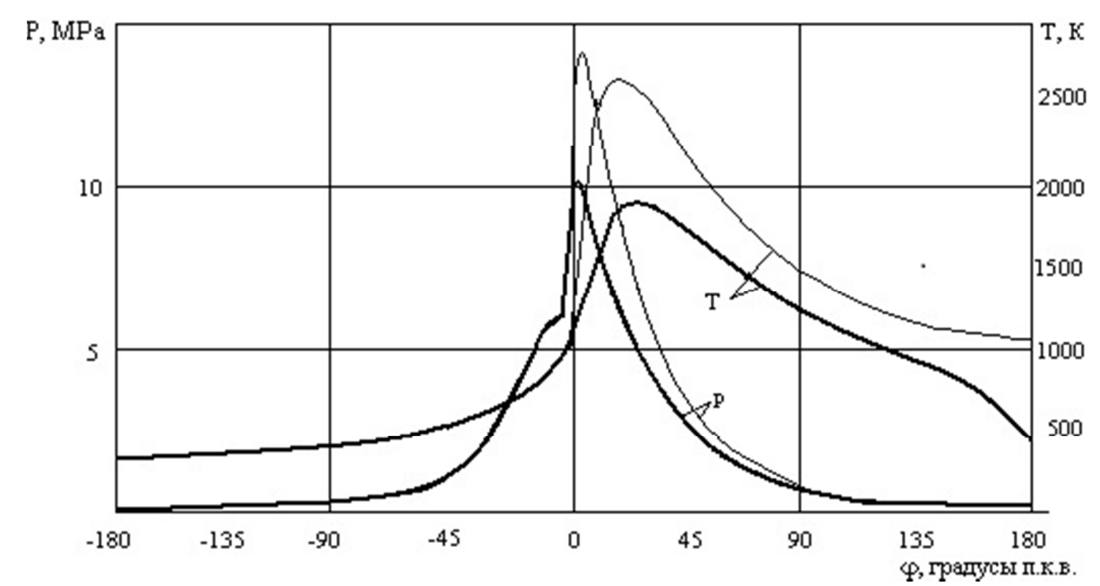

Figure 6. Diagrams of pressure $p$ and temperature $T$ of the ideal thermotropic cycle (fine lines) and the real (thickened lines) diesel cycle with supercharging $(\varphi$-crankshaft rotation angle).
The economic cycle of the engine by (18) provides at $\theta_{\eta}=$ 1,2 , which is not much more than the real value $\theta=1,07$, which corresponds to the regulation of the diesel engine for lower toxicity of exhaust gases. Calculations using (17) and (18) showed that a decrease in $\theta$ from 1.2 to 1.07 led to a decrease in $\eta_{\mathrm{t}}$ from 0.650 to 0,644 or slightly more than $1 \%$. This small value confirms the validity of the $\theta$ reduction method to reduce toxicity.

The thermotropic cycle generalizes the classical ideal cycle, the equations of which are obtained $A_{\mathrm{T}}=1, m=n$ (which transforms the thermotropic process into polytropic). For the isochoric cycle, we additionally have: $v_{\mathrm{d}} / v_{\mathrm{c}}=v_{f} / v_{\mathrm{c}}=1, p_{\mathrm{f}} / p_{\mathrm{d}}=\lambda$; for the isobaric: $v_{\mathrm{d}} / v_{\mathrm{c}}=1, v_{f} /$ $v_{\mathrm{c}}=\rho, p_{\mathrm{f}} / p_{\mathrm{d}}=1$; for the isochoric-isobaric cycle: $v_{\mathrm{c}} / v_{\mathrm{d}}=$ $1, v_{f} / v_{\mathrm{c}}=\rho, p_{\mathrm{f}} / p_{\mathrm{d}}=\lambda$.

In accordance with the considered model cycle scheme, the values of $m_{\mathrm{s}}=-2$ and $m_{\mathrm{r}}=5$ are obtained. However, the indicators of the cycle do not change, since combustion occurs almost only in the process of expansion.

\section{Conclusion}

1. The thermotropic ideal cycle generalizes three known at $p_{2}=p_{\max }, p_{1}=p_{\mathrm{d}}, \varphi_{2}=\varphi_{\text {pmax }}$ и $\varphi_{1}=\varphi_{\mathrm{d}}$. The maximum speed is determined for a smaller angle difference in the denominator on the right (20): $\varphi_{2}-\varphi_{1}=5$ degrees. The pressure and rotation angles of the shaft correspond to the points: for diesel engines $p_{2}=p_{\varphi \mathrm{d}+5}, p_{1}=p_{\varphi \mathrm{d}}$, and for engines with spark ignition $p_{2}=p_{c}, p_{1}=p_{\varphi c-5}$, where the indices $\varphi_{\mathrm{d}}$ and $\varphi_{\mathrm{c}}$ correspond to the angles at the points $d$ and $c$.,

Figure 6 shows real pressure and temperature diagrams for the tractor diesel with supercharged and calculated diagrams of the ideal thermotropic cycle obtained by formulas (6) and (7) of the initial data: $q_{0}=1.98 \mathrm{MJ} / \mathrm{kg} ; p_{\mathrm{a}}=0.143 \mathrm{MPa} ; T_{\mathrm{a}}=$ $362 \mathrm{~K} ; \theta=1.07 ; \varepsilon=16.5 ; \rho=8 ; m_{\mathrm{r}}=5 ; m_{\mathrm{s}}=-9$ [8]. The nature of the calculated dependences coincides with the diagrams of the real process, and the characteristic volumes correspond to the maximum pressures and temperatures. gas cycles: isochoric, isobaric, and mixed. This allows us to consider the model of one cycle instead of three: diesel engines, spark ignition engines and gas. A feature of the new model is the ability to analyze the impact on the cycle parameters of factors that are not taken into account in classical models: the law of heat dissipation in fuel combustion, the beginning and end of the combustion period, the pressure increase rate.

2. Thermotropic thermodynamic process generalizes classical gas processes: isochoric, isobaric, isothermal, adiabatic and polytropic. A special feature of the new model is the variable heat capacity of the process, the independence of the amount of heat of the process from the process parameters and the given law of heat exchange between the external source and the gas. The model provides much more opportunities for modeling and analysis of real physical processes in comparison with politropic.

3. The combined process "compression-expansion" by applying different values of the exponent processes compression and expansion allows to take into account in analytical calculations the main features of real combustion in engines with self-ignition or spark ignition.

4. A significant advantage of the new cycle model is 
mathematical simplicity. The exact integration of the initial system of equations allowed to Express the equations of processes and cycles in elementary functions. The accuracy of estimation of process and cycle parameters is quite high, since only traditional and physically based assumptions and approximations are included in the model.

\section{Acknowledgements}

The author thanks the head of the Department Professor Efros V. V. for the organizational and methodological assistance in testing the work as a teaching material for the bachelor's degree and co-authorship in publications on their promotion in related educational institutions.

\section{References}

[1] Vukalovich M. P., Novikov I. I. Engineering thermodynamics. Moscow-Leningrad, Gosenergoizdat, 1952, $567 \mathrm{p}$.

[2] Technical thermodynamics. Drugakov E. V., Kozlov N. I., Korneichuk I. K., etc. Under the editorship of Professor Krutov V. I. Textbook for technical colleges. Moscow, Higher school, 1971, $472 \mathrm{p}$.

[3] Zlobin V. G., Korotkova T. V., Technical thermodynamics. Textbook. Publishing house Spbgturp. 2011, 149 p. Il 52.

[4] Белов Г. В. Техническая термодинамика. Учебное пособие для академического бакалавриата. М., Издательство Юрайт. 2017. 252 р. ISBN 978-5-9916-9743-9.

[5] Bulidorova G. V., Galyametdinov Y. G., Yaroshevsky, H. M., Barabanov V. P. Physical chemistry. Book 1. Fundamentals of chemical thermodynamics. Textbook. Kazan., KSTU, 2011 218 p. - 978-5-7882-1151-0

[6] Orlin A. S. and others. Theory of working processes of piston and combined engines. Moscow, Publishing House "Mechanical engineering". 1971. $400 \mathrm{p}$.

[7] Kavtaradze R. Z. The theory of piston engines. Special chapter. Textbook for universities. M.: Izd-vo MGTU im. N. E. Bauman, 2008. 720 p.
[8] Internal combustion engine. In $3 \mathrm{kN}$. kN. 1. Theory of working processes. Textbook for high schools/ V. N. Lukanin, K. A. Morozov, A. S. Melikset khachiyan and others; Under the editorship of corresponding member. - cor. Ran, prof., Dr. Techn. Sciences V. N. Lukanina. M., Higher school, 2005. 479 p. ISBN 5-06-004142-5

[9] Stolbov M. S., Efros V. V. Analysis and mathematical description of the ideal thermotropic cycle engine. Bulletin of engineering. 2016, June. P. 82-86. ISSN 0042-4633.

[10] Yastrzhembskiy A. S. Thermodynamics and history of its development. Moscow-Leningrad, ed. "Energy", 1966, 667 p.

[11] Shule V. Technical thermodynamics. vol. 1, MoscowLeningrad, The main edition of the energy literature, 1935, $412 \mathrm{p}$.

[12] Stolbov M. S. Thermodynamic basis of heat release characteristics in combustion engine // XII Intern. scientific.prakt. Conf. "Fundamental and applied problems of perfection of piston engines". Vladimir: Vladimir State University, 2010. P. 240-245. ISBN 978-5-9984-0079-7.

[13] Stolbov M. S. Heat transfer from the gases to the walls of the cylinder of a tractor diesel engine with air cooling. // Proceedings of the NATI, number 198, Moscow, ONTI NATI, 1968. P. $39-79$.

[14] Vibe I. I.. New about the working cycle of engines. MoscowSverdlovsk, MASHGIZ, 1962, 271 p.

[15] Luzin N. N. Integral calculus. Moscow, State. publishing "Higher school", 1961. S. 416.

[16] Анго А. Математика для электро- и радиоинженеров, М., Изд-во «Наука», Гл. редакция физ.-мат. литературы, 1967. C. 780 .

[17] Lungu K. N., Makarov E. V. the Higher mathematics. Textbook. Part 1, Moscow, Fizmatlit, 2013 - p. 217 - 978-59221-1500-1.

[18] Stolbov M. S. Thermotropic gas processes and cycles. LAP LAMBERT Academic Publishing RU, 2018, p. 121. ISBN 978-613-7-75075-9.

[19] Inozemtsev N. V., Koshkin V. K. Combustion Processes in engines. Moscow, MASHGIZ, 1949, p. 344. 